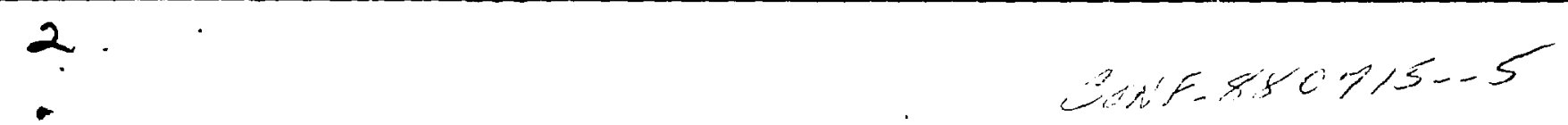

BROOKHAVEN NATIONAL LABORATORY

June, 1988

BNL -41469

BNL -41469

DE88 015195

\title{
Soft Mode Behavior in Ni-Al Alloys
}

S. M. Shapiro, B. X. Yang, G. Shirane, J. Z. Larese

Brookhaven National Laboratory Upton, NY 11973, U. S. A.

\author{
L.E.Tanner \\ Lawrence Livermore National Laboratory Livermore, CA 94550, U.S.A. \\ and \\ S.C.Moss, \\ University of Houston, Houston, TX 77004, U.S.A.
}

\begin{abstract}
Inelastic neutron scattering experiments performed on carefully prepared single crystals of $\mathrm{Ni}_{x} \mathrm{Al}_{1-x}(\mathrm{x}=50,58,62.5$ at. percent) reveal an anomaly in the [550]-TA mode whose position in 5 depends linearly on $\mathbf{x}$. The temperature dependent studies of the $62.5 \%$ alloy show marked softening of the phonon energy at $\zeta=1 / 6$. At the same temperatures, an elastic central peak develops. At $\mathrm{T}_{M}=80 \mathrm{~K}$ a new structure develops which exhibits a modulation at nearly,but not exactly, $\zeta=1 / 7$.
\end{abstract}

Key Words: Soft modes, Martensitic Transformations 
Martensitic phase transitions have been extensively studied by metallurgists over the past 100 years[1]. Physicists have recently become interested in these systems. There is substantial effort to apply many of the methods that have been developed over the last 20 years in the study of nearly second order phase transitions in ideal materials such as the perovskite ferroelectrics (e.g. $\mathrm{BaTiO}_{3}$ ) or antiferroelectrics (e.g. $\mathrm{SrTiO}$ ). The soft mode concept has proven very successful in describing these phase transitions and predicting the low temperature structure. Martensitic transitions, however, are first order phase transitions and most do not exhibit extensive phonon softening[2!. On the other hand, most do exhibit phonon anomalies which are not obviously related to the low temperature structure[3].

The alloy $\mathrm{Ni}_{x} \mathrm{Al}_{1-x}$ is a material extensively studied because of its martensitic properties[4]. The high temperature phase of $\mathrm{Ni}_{x} \mathrm{Al}_{1-x}(50<x<63$ at.\%) has the $\mathrm{CsCl}$ or $\beta$ structure $(a=2.858 \dot{A})$ where the excess $\mathrm{Ni}$ is randomly distributed over the $\mathrm{Al}$ sites. For $x>60$ at.\% a phase transition occurs for $T_{M}>0$ to a low temperature structure which is not yet fully determined. In this brief report we present results on the precursor effects observed in the phonon behavior of $\mathrm{Ni}_{z} \mathrm{Al}_{1-z}$. All experiments were performed on the 3-axes instruments at Brookhaven National Laboratory's High Flux Beam Reactor.

A previous study at room temperature shows that the [ 550$]-\mathrm{TA}$ mode (displacements along [1 $1 \overline{1} 0]$ ) exhibits an anomaly at finite $S[5]$. This mode corresponds to the elastic constant $c^{\prime}=1 / 2\left(c_{11}-c_{12}\right)$ in the limit $s \rightarrow 0$. The position of the anomaly varied linearly with $x$ and for $x=62.5$, the position is at $S \approx 1 / 6$. The temperature dependent study of this alloy shows a significant change with temperature. Figure 1a gives the temperature dependence of this dispersion curve for $T>T_{M} \approx 80 K$. It is clearly seen that the frequency softens as $\mathrm{T} \rightarrow \mathrm{T}_{M}$ and the anomaly at $\zeta=0.16$ deepens. The elastic scattering along this same direction is shown in Figlb. At room temperature there is a weak shoulder at $\zeta \approx 1 / 6$ and this intensity grows as $\mathrm{T} \rightarrow \mathrm{T}_{M}$. At $\mathrm{T}=85 \mathrm{~K}$ a well defined broad peak is present with a full-width-at-half-maximum $\Delta S=0.056 a^{*} \sqrt{2}$ corresponding to a correlation range of $6 \dot{A}$. This peak in the diffuse scattering occurs at the same $s$ value as the position of the minimum in the dispersion curve and has the characterisitics of a central peak observed in other structural phase transitions[6]. 
The temperature dependence of the phonon energy at $\zeta=0.16$ and the central peak intensity at the same wavevector are shown in Fig.2. In Fig. 2a the phonon energy $(\hbar \omega)^{2}$ is plotted as a function of temperature. A straight line is observed which extrapolates to $\hbar \omega=0$ at a negative temperature $T=-30 \mathrm{~K}$. This is consistent with the behavior of a first order phase transition occurring at $T_{M} \approx 80 \mathrm{k}$. (The actual phase transition depends on the rate of cooling since there is an inherent time dependence for the sample to reach equilibrium). The behavior of the elastic intensity at $\zeta=0.16$ is plotted in Fig.2b as $\mathrm{I}^{-1}$ vs $T$. The curve is not a straight line. Below $T=80 \mathrm{~K}$ there is an upswing in $\mathrm{I}^{-1}$ which is due to a coexistence of the high and low temperature phases. The intensity of the central peak becomes less because a smaller fraction of the sample remains in the high temperature phase as the material is cooled further.

The study of the cubic phase of $\mathrm{Ni}_{x} \mathrm{Al}_{1-z}$ in many respects looks similar to other soft mode systems: there is a softening of a phonon at a particular wavevector and this softening is accompanied by a central peak at the same wavevector. The $\mathrm{Ni}_{x} \mathrm{Al}_{1-x}$ system is ideal for studying the central peak because the soft mode remains underdamped and well separated from the central peak over the entire range of softening. The differences in the shape of the curves in Fig. 2 shows that the central peak intensity varies faster than $\left(\hbar \omega_{s}\right)^{-2}$. In fact, the data for $\mathrm{T}>85 \mathrm{~K}$ is consistent with the intensity of the central peak varying as $I \sim\left(\hbar \omega_{s}\right)^{-4}$. This power law is exactly what was proposed if the origin of the central peak was a result of Huang scattering due to defects[7].

A companion electron diffraction study on a sample of $\mathrm{Ni}_{62.5} \mathrm{Al}_{37.5}$ cut from the same boule shows features similar to Fig $\mathrm{Ib}$ at several Bragg peaks [8]. The high resolution image of the diffraction pattern exhibits an underlying $13 \dot{A}$ periodic modulation of the atomic spacing along the [110] direction with displacements along [110]. These visible distortions of the cubic lattice create a strain field which is the origin of the central peak observed in Fig. $1 \mathrm{~b}$.

The question remains as to the relationship between these precursor effects and the low temperature structure. $\mathrm{X}$-ray and electron diffraction studies of this alloy show that there is a 7 -fold modulation along the $[110]$ direction in the martensitic phase $[8,9]$. The value of $1 / 7=0.143$ is reasonably close to 0.16 . Higher order terms in the free energy describing the system could be responsible for having a $1 / 7$ modulation of the low temperature 
structure even though the precursor effects appear at $1 / 6$. A recent high resolution neutron diffraction study of the low temperature phase showed that the modulation is more complicated than a simple sine wave with wavevector of $1 / 7$ of the host reciprocal lattice vectors [10]. The spacing between the peaks is not exactly $1 / 7$ nor is the spacing the same from peak to peak. Also, the linewidths of the satellites are larger than the instrumental resolution and vary from peak to peak. The spectra have a distinct similarity to a Hendricks-Teller lineshape observed in intercalated graphite and other layered structures with disorder[11]. This similarity suggests that stacking faults of the closed-packed [110] planes play an important role in the low temperature structure. These effects are currently being studied.

The authors gratefully acknowledge the many discussions with R. Gooding and J.A.Krumhansl. Work at BNL and LLNL was performed under the Division of Materials Sciences, U.S. Department of Energy under contracts, DE-AC02-76CH00016 and W-7405-ENG-48, respectively. Work at Houston supported by NSF Grant No. DMR8603662. 


\title{
REFERENCES
}

1. Z. Nishiyama Martensitic Transformations (Academic Press, New York, 1978).

2. J. A. Krumhansl in Competing Interactions and Microstructures: Statics and Dynamics, ed. by R. LeSar, A. Bishop, and R. Heffner (SpringerVerlag, New York, 1980) p.50.

3. S. M. Shapiro in ref. 2. p. 84.

4. I. M. Robertson and C. M. Wayman, Phil. Mag. 48 (1983) 421.

5. S. M. Shapiro, J. Z. Larese, Y. Noda, S. C. Moss and L. E. Tanner, Phys. Rev. Letters 57 (1986) 3199.

6. A. D. Bruce and R. A. Cowley, Structural Phase'Transitions (Taylor \& Francis London 1981).

7. J. D. Axe S. M. Shapiro and G. Shirane, Anharmonic Lattices, Structural Transitions and Melting, ed. by T. Riste (Nordhof, Leiden 1974) p. 23.

8. L. E. Tanner in ref. 2. p. 74.; L. E. Tanner, A. R. Pelton, G. Van Tendeloo, D. Schryvers, M. Wall. Scripta. Met. (to be published).

9. V. V. Martynov, K. Enami, L. G. Khandros, S. Nenno, A. V. Tkachenko Phys. Met. Metall. 55 (1983) 136.

10. S. M. Shapiro, B. X. Yang, G. Shirane and L. E. Tanner (to be published); G. Shirane, Proceedings of the MRS Meeting, Tokyo, 1988 (to be published).

11. S. Hendricks and E. Teller, J. Chem. Phys. 10 (1942) 147.

\section{DISCLAIMER}

\begin{abstract}
This report was prepared as an account of work sponsored by an agency of the United States Government. Neither the United States Government nor any agency thereof, nor any of their employees, makes any warranty, express or implied, or assumes any legal liabi.ity or responsibility for the accuracy, completeness, or usefulness of any information, apparatus, product, or process disclosed, or represents that its use would not infringe privately owned rights. Reference herein to any specific commercial product, process, or service by trade name, trademark, manufacturer, or otherwise does not necessarily constitute or imply its endorsement, recommendation, or favoring by the United States Government or any agency thereof. The views and opinions of authors expressed herein do not necessarily state or reflect those of the United States Government or any agency thereof.
\end{abstract}




\section{FIGURE CAPTIONS}

Figure 1. (a) The temperature dependence of the dispersion of the [550]-TA mode. (b) The temperature dependence of the elastic scattering along the [ $5 \zeta 0]-T A$ direction.

Figure 2. (a) $(\hbar \omega)^{2}$ vs. $T$ for the [550]- ta mode for $\zeta=0.16$. (b) $I^{-1}(\hbar \omega=0)$ vs $\mathrm{T}$ for the elastic scattering along the [550]-TA direction for $\varsigma=0.16$. 


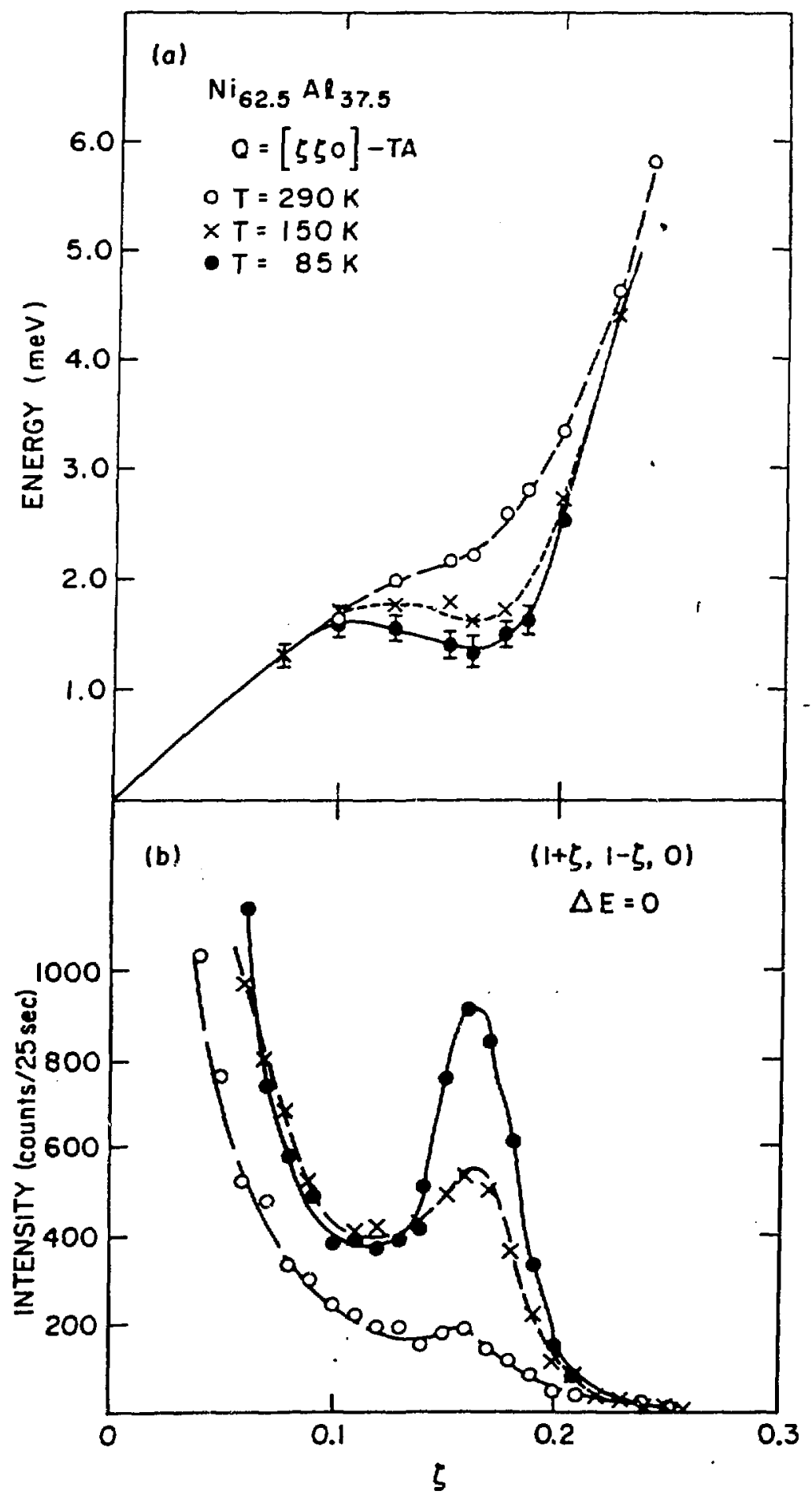

FIGIRE 1 


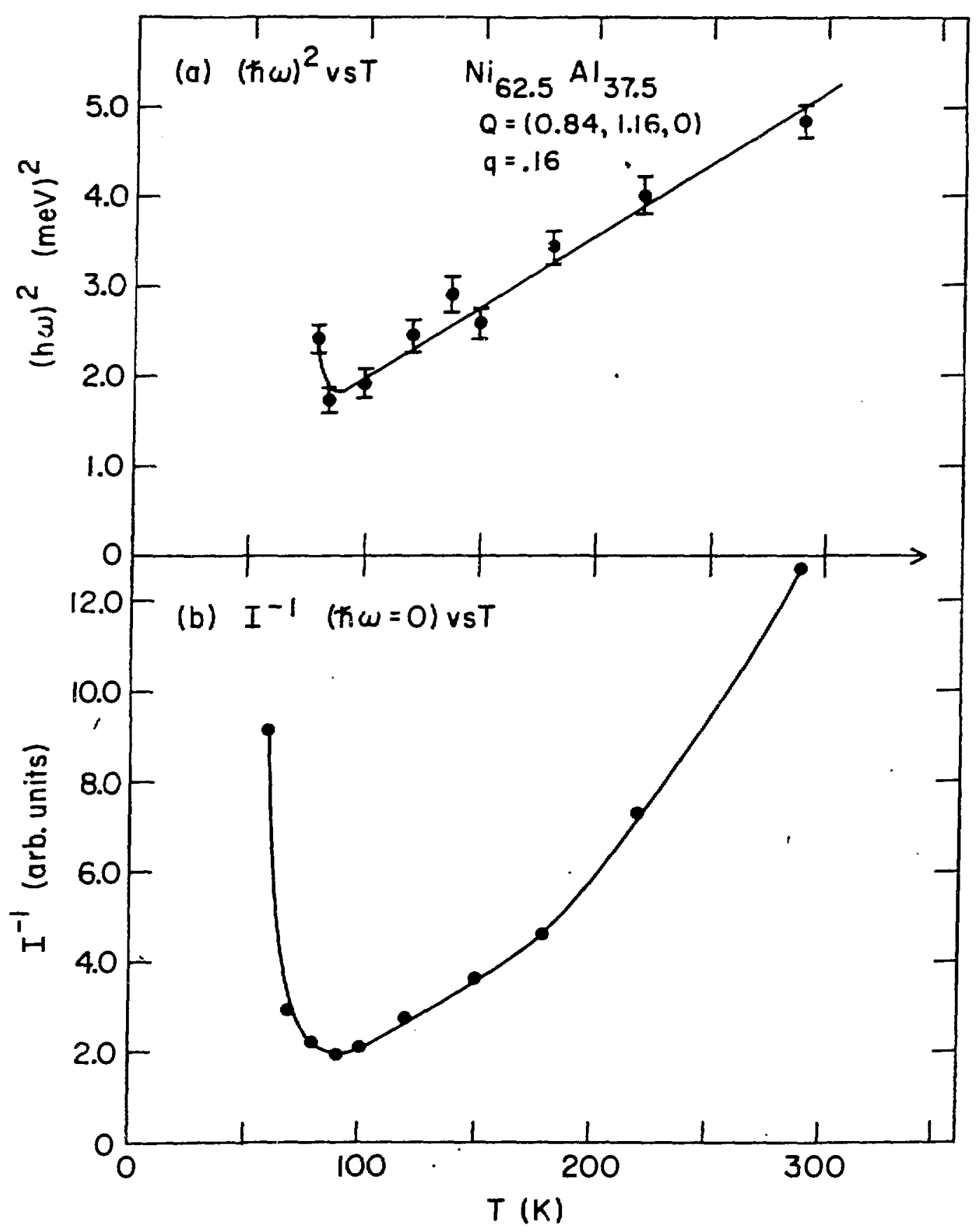

